\title{
Challenges in design process of gear micropump from plastics
}

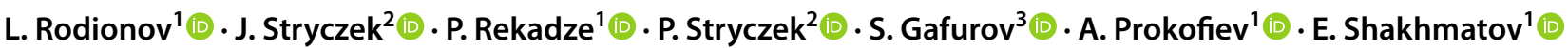

Received: 12 June 2020 / Revised: 4 December 2020 / Accepted: 9 December 2020 / Published online: 18 February 2021

(c) The Author(s) 2021

\begin{abstract}
Plastics are more and more frequently applied in the construction of hydraulic components and systems, and recently, in the building of microhydraulic assemblies. The basic unit of the microhydraulic systems is a micropump. It is an object which is quite difficult to design and manufacture, especially when using plastics. The paper is a presentation of challenges that must be met in the process of designing gear pumps, and, at the same time, of the theoretical grounds for the designing of those objects. The challenges include the geometry and the gears, the construction of the pump body, the hydraulics of the pump, and the material - the plastic of which the pump is made. The knowledge on those issues was applied to make and to successfully test a prototype of a micropump.
\end{abstract}

Keywords Gear micropumps $\cdot$ Plastics $\cdot$ Design process $\cdot$ Fluid power

\begin{tabular}{ll} 
Abbreviations \\
$\alpha$ & Involute tooth profile angle \\
$\delta$ & Irregularity of delivery coefficient/coefficient \\
& of pulsation delivery \\
$\eta_{v}$ & Volumetric efficiency \\
$\eta_{\mathrm{a}}$ & Total efficiency \\
$\varepsilon$ & Strain \\
$\rho$ & Apparent friction angle \\
$\sigma$ & Stress \\
$q$ & Specific delivery/delivery per one revolution \\
$Q_{t}$ & Theoretical delivery \\
$Q$ & Screw force \\
$a$ & Teeth centers distance \\
$A$ & Water absorption \\
$b$ & Tooth width \\
$d$ & Pitch diameter \\
$d_{a}$ & Addendum diameter \\
$d_{f}$ & Dedendum diameter \\
$d_{s}$ & Tread diameter \\
$E$ & Young modulus \\
$F$ & Force \\
& \\
\hline
\end{tabular}

J. Stryczek

jaroslaw.stryczek@pwr.edu.pl

1 Samara National Research University, Samara, Samara Region, Russia

2 Wroclaw University of Science and Technology, Wrocław, Lower Silesia, Poland

3 Innopolis University, Innopolis, Tatarstan, Russia

$\begin{array}{ll}D & \text { Inlet, outlet diameter } \\ G_{A}, G_{R} & \text { Axial, radial clearance } \\ \Delta G_{A}, \Delta G_{R} & \text { Increase of axial and radial clearance } \\ h a^{*}=y & \text { Involute gear tooth depth ratio } \\ L e q & \text { Level of noise } \\ m & \text { Modulus } \\ M & \text { Screw torque } \\ n & \text { Rotational speed } \\ p & \text { Pressure } \\ R_{e} & \text { Yield strength } \\ S & \text { Shrinkage } \\ t & \text { Temperature } \\ T & \text { Torque } \\ W & \text { Linear elongation } \\ x & \text { Correction coefficient } \\ x_{g} & \text { Boundary correction coefficient } \\ z & \text { Number of teeth } \\ z_{g} & \text { Boundary number of teeth }\end{array}$

\section{Introduction}

There is an increasing trend towards the use of plastics in mechanical engineering. More and more new plastics and composites are designed and manufactured, which meet high strength and operational requirements. Those materials are then subjected to intensive tests concerning friction [1], wear [2], and operation in various conditions such as the sea water [3]. Plastics are successfully utilized to build 
machine components and assemblies. They are, among others, applied for the construction of slide bearings [4] and rolling bearings [5] as well as gears [6]. The damping properties of plastics make gears work with low noise [7] and the selection of an appropriate material ensures smooth collaboration of the teeth [8]. Plastics are also utilized for the making of machine bodies [9].

Plastics have also been introduced in the construction of hydraulic components and systems [10]. They have been applied, for example, in the building of both an oil pump for a motorcycle engine made of PPS and PTFE [11] and gerotor gears [12], gerotor pumps [13], and gear pumps [14]. Also, hydraulic valves, such as relief valves [15] and on/off valves [16], as well as hydraulic cylinders [17] have been successfully made of plastics. They have also found application in various industries such as engineering, automation, and robotics [18]. Plastics have been used as a material for valve block bodies [19], in which, thanks to the application of injection technology, it was possible to make a complicated system of internal shapes.

The analysis of the presented works shows that the utilization of plastics offers various benefits, including:

- construction (weight reduction, damping of vibration and noise, reduction of friction, improvement of tribological properties, possibility of self-sealing, possibility of working with water, and chemically aggressive agents); - technological (possibility of producing complicated shapes using a simple method of injection modelling, simplification, and shortening of the production process).

The application of plastics is also accompanied by adverse features such as: reduced strength, lower dimensional stability, and resistance to higher temperatures. Unfavourable features can be partially compensated by the selection of more and more durable materials and special design solutions of a hydraulic element.

Plastics are now entering a special field of hydraulics, namely micro-hydraulics. The basic unit of the microhydraulic system is a micropump. The paper [20] shows that there are possibilities of using plastics for the construction of micropumps.

When designing plastic micropumps, works on metal gear micropumps [21] as well as work [22] on obtaining high efficiency of micropumps can be referred to. Also, [23] should be taken into account, where new solutions for bearing nodes in micropumps were presented. During the design process, it is also necessary to consider broader works on the modeling and simulation of flows in gear pumps [24] and gerotor pumps [25] as well as the simulation of the phenomena occurring between the mechanical assemblies and the working medium in those machines [26]. The group also includes research works on flow pulsation [27], and modeling of that pulsation [28]. The subject of plastic micropumps is also encouraged by pilot studies [29], showing that the use of plastics in gear pumps reduces noise.

Those and other works, however, do not exhaust the problem in both its scientific and technical aspect. Considering that, the following aims of the work were defined:

- formulation of the challenges to be met in the process of designing plastic micropumps, which is also the formulation of the theoretical grounds for the design of those elements;

- structural design, manufacturing, and experimental verification of the micropump, which would prove the design process of the micropump core.

It is expected that the experience gained in this project will serve the development of other micro-hydraulic components such as valves or cylinders.

\section{Design, hydraulic, and material challenges}

Three fundamental challenges will be taken up during the design process of plastic micropumps:

1. The design challenge- how to design a unit featuring small dimensions and weight, simple construction, and a small number of parts;

2. Hydraulic challenge- how to ensure low value of special delivery rate $q\left[\mathrm{~mm}^{3} / \mathrm{rev}\right]$, low value of irregularity of delivery $\delta$ [\%], a possibly of high operating pressure $p[\mathrm{MPa}]$, and high volumetric efficiency $\eta_{\nu}[\%]$;

3. Material challenge - what materials and technologies should be applied to build a plastic micropump.

A schematic structure of the micropump is shown in Fig. 1. It can be divided into three main systems, i.e., a system of gears (1) with shafts and a system of the body (2).

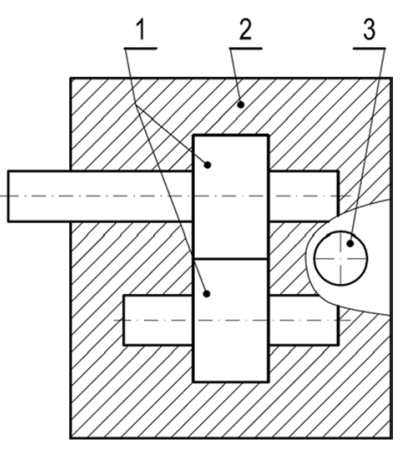

Fig. 1 Schematic structure of the micropump: 1-sub system of the gears; 2 -body; $\mathbf{3}$-subsystem of the channels and clearances 
The system of the body includes a system of internal channels and clearances (3) which gears co-operate with, carrying out the pumping process.

Therefore, the design challenges related to both the above-mentioned key systems and the systems of the micropump have been analyzed.

\subsection{The gear system}

To ensure a small size of the micropump and its low delivery rate, gears with as small dimensions as possible should be designed. At the same time, the gears should have high strength to work under high mechanical (torque, intertooth forces) and hydraulic (working pressure) loads. Based on [30], the involute tooth profile with a typical angle $\alpha=20^{\circ}$ is assumed. To obtain small dimensions, a low value of the tooth module $m<1$ and normal teeth featuring the tooth depth ratio of $h a^{*}=y=1$ are adopted. To ensure enough strength of the teeth of the small size, the teeth must be strengthened at their roots. Therefore, two cases presented in Fig. 2 ought to be considered.

In the first case (Fig. 2a), when the number of teeth lower than the boundary number of teeth is used, that is:

$z_{1}=z_{2}<z_{g}=15$,

the teeth are undercut. In this situation (Fig. 2b), it is necessary to introduce the tooth profile correction $\mathrm{P}$ with the gear axes moved apart and correction coefficients:

$x_{1}=x_{2} \geq x_{g}=\frac{2 y}{\sin ^{2} \alpha}$.

In the second case (Fig. 2c), when the number of teeth equal or higher than the boundary number is used, that is:

$z_{1}=z_{2} \geq z_{g}=15$,

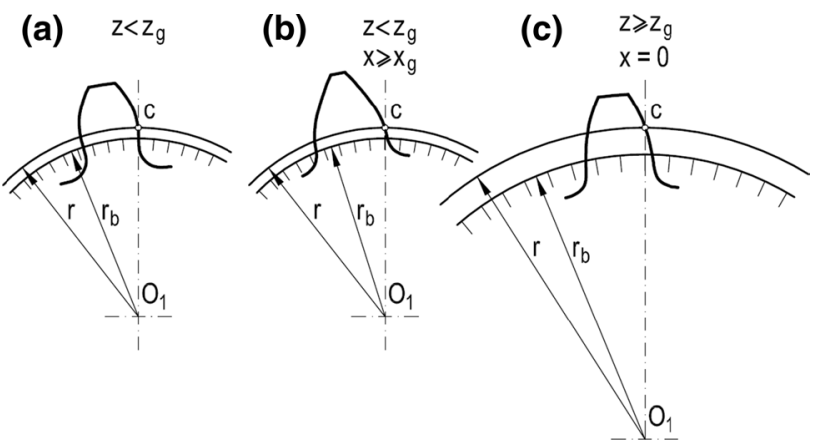

Fig. 2 Shaping involute teeth: a $z<z_{g}, x>=x_{g}$, no $P$-correction, teeth undercut. $\mathbf{b} z<z_{g}, x=0, P$-correction, teeth not undercut. $\mathbf{c} z>=z_{g}$, $x=0$, no $P$-correction, teeth not undercut there is no undercutting of the teeth, and the correction can be used to further strengthen the tooth. In both cases, the gear dimensions increase, but at a small module $m<1$ and the tooth depth ratio $y=1$, the gear dimensions are not excessively increased. The utilizing of the second case, i.e., the increased number of teeth $z_{1}=z_{1} \geq 15$ provides additional benefits, as it reduces the value of the delivery irregularity coefficient $\delta$ and the noise of the pump operation. It is recommended that both gears have the same number of teeth $z_{1}=z_{2}=z$ to ensure the symmetrical structure of the pump body. It is also assumed that the gears should be made together with shafts, because then they can rotate in the bearing seats made directly in the body of the pump [22] That simplifies the design solution of the micropump and reduces the number of parts. The gear width $b$ is derived from the performance formula (1) given later in this paper.

\subsection{The system of the body}

Small dimensions of the gears may guarantee small body dimensions. At the same time, the body should have high strength, so that it could carry mechanical and hydraulic loads, and be characterized by stability of the shape and dimensions to ensure internal tightness of the micropump.
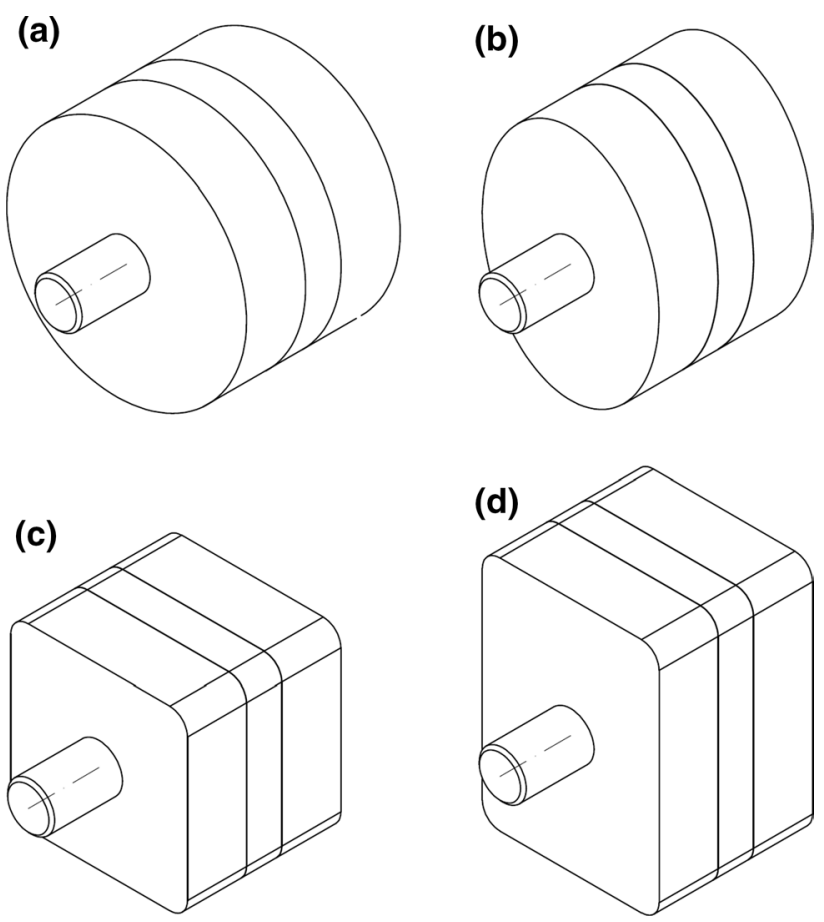

Fig. 3 Typical shapes of gear pump bodies. a Cylindrical body with a round cross-section. b Cylindrical body with an oval cross-section. $\mathbf{c}$ A prism-shaped body with a square cross-section. d A prism-shaped body with a rectangular cross-section 
Figure 3 shows after [31] that two basic body shapes can be distinguished:

- the cylindrical body of a circular or oval cross-section (Fig. 3a, b),

- the prism-shaped body of a square or rectangular crosssection (Fig. 3c, d).

The body of the housing should be divided into parts (plates). Mostly, there are three parts which can be distinguished in the structure. In the central part, there is a system of gears, while on its side plates, there are bearing seats and a system of internal channels. The three plates are bolted together, which ensures both a proper clamping between the plates and the rigidity of the entire body.

\subsection{The system of channels and internal clearances}

A system of channels and clearances is formed in the body of the pump, which ensures the inflow and outflow of the working medium to the rotating gears, as shown in Fig. 4 and according to [26].

As the dimensions of the gears are small, the diameters (cross-sections) of the channels are also small.
Fig. 4 Subsystem of internal channels and clearances in the gear micropump. a Simple system, b Angular system: 1body, 2-system of channels $C L_{I}, C R_{I}, B_{I}$-inlet channel, chamber and bridge $C L_{O}, C R_{O}$, $B_{O}$-outlet channel, chamber and bridge $G_{A}, G_{R}$-axial and radial clearance
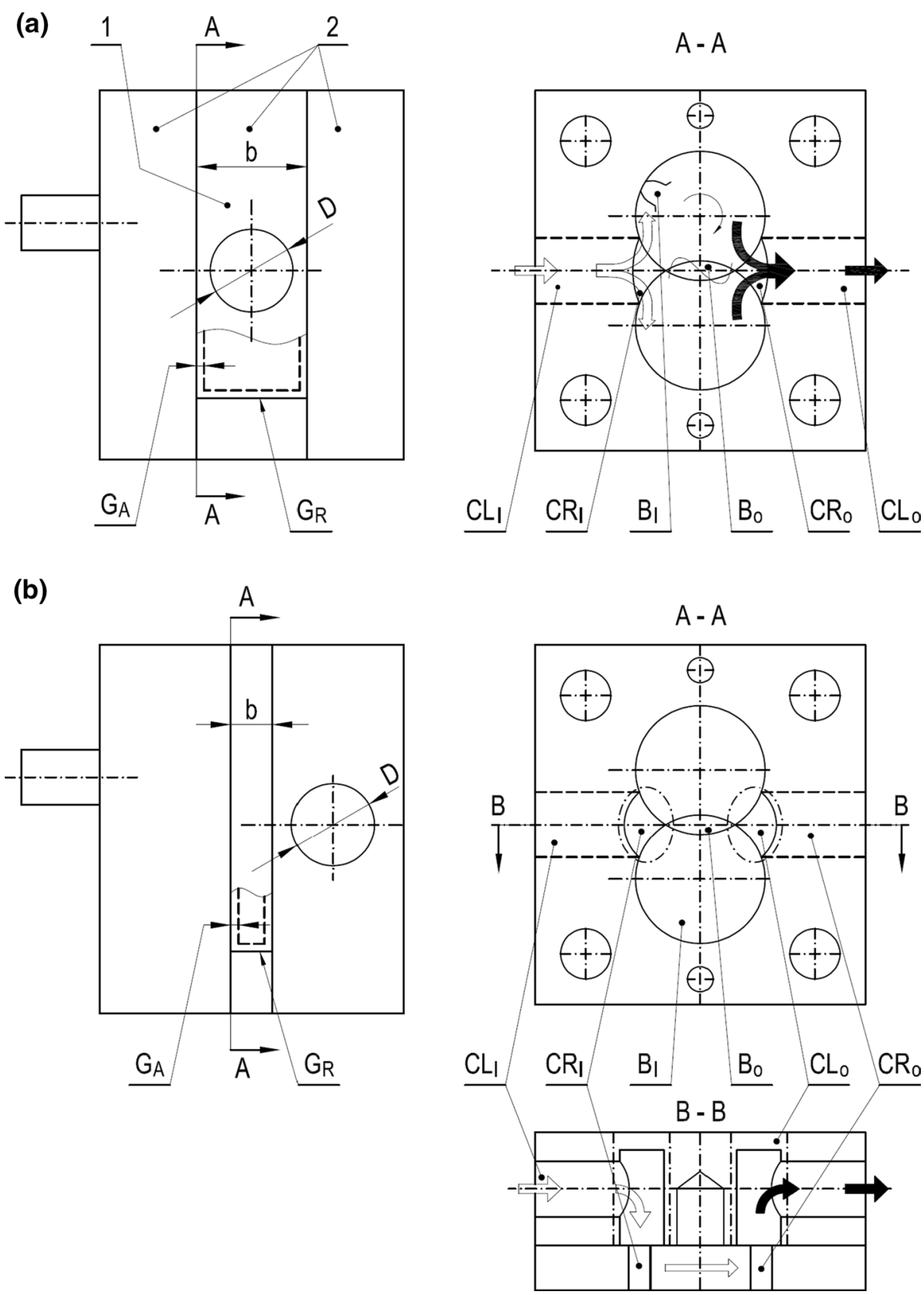
The arrangement (architecture) of the channels depends on the diameter $D$ of the connection holes and the width of the central body $b$. If the diameter of the hole $D$ is smaller than the width $b$, then the entire system of channels can be located in the central body (see Fig. 4a, cross-section A-A). This is called a simple system of channels. It consists of the $C L_{I}$ inlet channel, $C R_{I}$ inlet chamber, and $B_{I}$ inlet bridge, which are used to deliver the working medium into the pump. From the inside of the pump, the working medium is discharged through the $C R_{O}$ outlet chamber and $C L_{O}$ outlet channel. The $\mathrm{B}_{\mathrm{O}}$ outlet bridge separates the inlet (suction) from the outlet (discharge) zones. If the diameter of the connection hole $D$ is larger than the width of the central body $\mathrm{b}$, then the entire system of channels cannot be placed in the central body. The channels must also be arranged in the rear body (see Fig. 4b, cross-sections A-A, B-B). This is called the angular system of channels. The $C L_{I}$ input and $C R_{O}$ output channels are located in the rear body in parallel (B-B cross-section, solid line) or perpendicular (B-B crosssection, dotted line) to the central body. Those channels are connected with the $C R_{I}$ and $C R_{O}$ chambers located in the central body. When shaping the arrangement of the internal
This formula has a form which is favorable for the design practice as it comprises the hydraulic parameter, i.e., unit specific delivery $q$, with the predetermined meshing parameters $z, m, b, x, y, \alpha$. Thus, it is easy to indicate the influence of the meshing parameters on the pump performance. In practice, this formula can be used in two ways:

- the unit specific delivery rate $q$ and the number of teeth $z$ are assumed and the gear width $b$ is calculated,or

- the number of teeth $z$ and the gear width $b$ are assumed and the unit specific delivery rate $q$ is determined.

The theoretical delivery rate $Q_{t}$, without taking into account the volumetric losses, is obtained by multiplying the specific delivery rate $q$ by the rotational speed $n$ of the pump's shaft, namely:

$Q_{t}=q n$.

The unevenness of the delivery rate $\delta$ (or delivery pulsation) is also determined on the basis of [30] using the formula:

$\delta=\frac{\left(1+\frac{z_{1}}{z_{2}}\right) \pi^{2} \cos ^{2} \alpha_{0}}{4\left[\left(\frac{z_{1}}{2}+x_{1}+y_{1}\right)^{2}+\frac{z_{1}}{z_{2}}\left(\frac{z_{2}}{z}+x_{2}+y_{2}\right)^{2}-\frac{z_{1} \cos ^{2} \alpha_{0}}{4 \cos ^{2} \alpha_{w}}\left(z_{1}+z_{2}\right)-\left(1+\frac{z_{1}}{z_{2}}\right) \frac{\pi^{2} \cos ^{2} \alpha_{0}}{12}\right]}$.

flow channels, it is necessary to ensure the lowest possible flow resistance. $G_{A}$ and radial $G_{R}$ clearances should be optimized due to the internal hydraulic tightness, mechanical resistance of the gears' movement, and the lubrication possibilities.

Hydraulic challenges involve mainly the calculating and designing of the hydraulic parameters of the micropump. Those calculations are related to three basic parameters, i.e., delivery rate (unit specific delivery $q$ and theoretical delivery $Q_{t}$ ), delivery pulsation $\delta$, and efficiency (volumetric efficiency $\eta_{v}$ and total efficiency $\eta$ ). The unit specific delivery rate is derived from the formula given in [26] in the form:

$$
\begin{aligned}
q= & \pi b m^{2}\left[\left(\frac{z_{1}}{2}+x_{1}+y_{1}\right)^{2}+\frac{z_{1}}{z_{2}}\left(\frac{z_{2}}{z}+x_{2}+y_{2}\right)^{2}\right. \\
& \left.-\frac{z_{1}}{4} \frac{\cos ^{2} \alpha_{0}}{\cos ^{2} \alpha_{w}}\left(z_{1}+z_{2}\right)-\left(1+\frac{z_{1}}{z_{2}} \frac{\pi^{2} \cos ^{2} \alpha_{0}}{12}\right)\right] .
\end{aligned}
$$

The critical parameter affecting the pulsation of delivery $\delta$ is the number of teeth $z$. The higher the number of teeth $z_{1}$, $z_{2}$, the smaller the pulsation of the delivery rate.

Although the delivery rate $q$ and the delivery pulsation $\delta$ can be calculated, the maximum working pressure $p$, the volumetric efficiency $\eta_{v}$ and the total efficiency $\eta$ cannot be calculated. The values of those parameters can only be predicted. Generally, they depend on the internal tightness of the pump, which is characterized by the axial clearance $G_{A}$ and the radial clearance $G_{R}$.

The axial clearance $G_{A}$ is formed while assembling the pump between the side surface of the gears and the side surface of the central body (Fig. 4). During the pump operation, the side bodies get deformed and the clearance gets enlarged by the value $\Delta G_{A}$. Total $G_{A}+\Delta G_{A}$ clearance should not be larger than the allowable axial clearance $G_{A}$ allow, that is:

$G_{A}+\Delta G_{A} \leq G_{A \text { allow }}$.

A similar situation is with the radial clearance $G_{R}$, which is formed while assembling the pump between the top flanks of the gears and the seat in the central body (Fig. 4). The central body also gets deformed and this clearance gets enlarged by the value $\Delta G_{R}$. Also in this case, the total 
clearance should not be larger than the allowable radial clearance, namely:

$G_{R}+\Delta G_{R} \leq G_{R \text { allow }}$

The increase in the clearances $\Delta G_{A}$ and $\Delta G_{R}$ can be eliminated using the axial and radial clearance compensation systems. They ensure high internal tightness of the pump in the entire operating range and guarantee high operating pressure $p$ and efficiency $\eta_{\nu}$ and $\eta$.

The lack of a compensation system can be partially replaced by the use of a higher strength material (plastics), a special body shape, e.g., an asymmetrical body [31], and the connection of the body plates tightened with screws. Obviously, the exact manufacture of the body and gears, as well as their proper assembly, are always fundamental. Finally, the working pressure values $p$ and the efficiency $\eta_{v}$ and $\eta$ result from experimental tests of the pump prototype.

\subsection{The material challenge}

The material challenge is a basic challenge in the process of designing a micropump. Plastics intended for the construction of micropumps should meet the following criteria:

1. high strength, i.e., high yield strength $R_{\mathrm{e}}$ and large Young's modulus;

2. dimensional stability, i.e.,

- small shrinkage $S$ resulting from the injection molding process,

- small linear elongation $W$ due to an increase in temperature $t$ of the working medium and in the ambient temperature,

- low water absorption $A$ in the working medium;

3. the possibility of easy machining and plastic forming as well as the lowest possible price and market availability.

Special attention should be paid to the strength analysis of the material. This was considered using Fig. 5 which presents a stress-strain diagram for four selected materials: structural steel and plastics such as PEEK, PPS, and POM.

The characteristics of the plastics were developed based on own research $[13,31]$. The figure shows that the steel has a sharp yield point $R_{\mathrm{e}}$, which separates the elastic from the plastic areas. Plastics, however, do not have a sharp yield point; therefore, the concept of proof stress $R_{\mathrm{e}} 0.2$, known from the field of strength of materials, can be used. The $R_{\mathrm{e} 0.2}$ point means that at this stress, there is a permanent elongation of the sample equal to $0.2 \%$ of its length. In Fig. 5, the $R_{\mathrm{e} 0.2}$ point is marked for plastics. Theoretically, this means that the materials working in the range of deformations of up to 0.2 remain in the elastic range. This in turn means that after loading and then unloading, the material should return

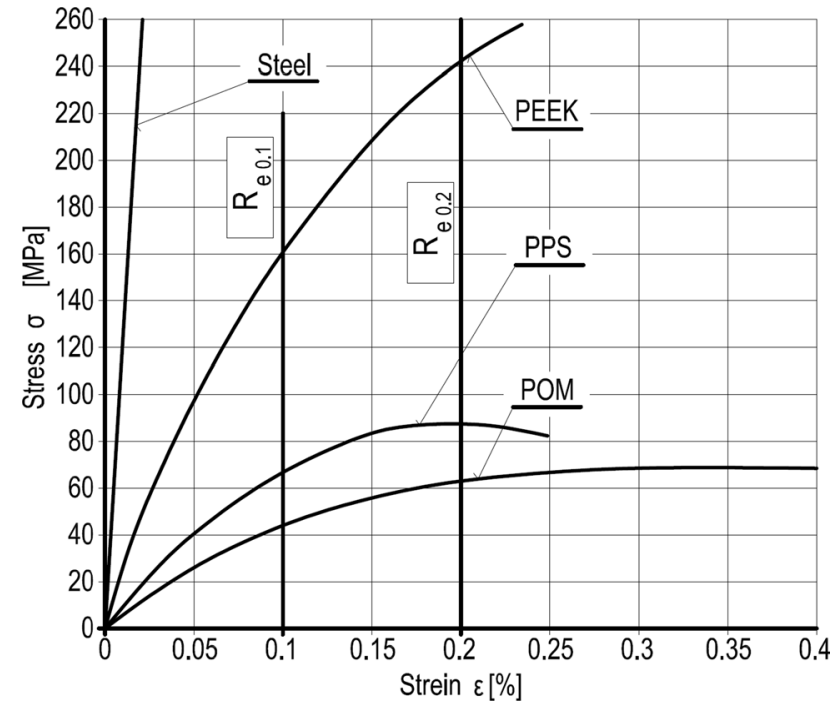

Fig. 5 Stress-strain diagram for different materials: steel, PEEK, PPS, and POM

to its original shape. Experiments show [31] that POM, for instance, under repetitive loading and unloading, and at temperature $t$, loses its strength and the yield point $R_{\mathrm{e} 0.2}$ gets significantly reduced. In this situation, a challenge arises consisting in the establishing of a lower proof stress of the material. It is proposed to take it by half and set it as $R_{\mathrm{e} 0.1}$. It can be assumed that adopting it in the $0.1 \%$ strain range will allow to maintain the elastic nature of the work of the material utilized in the micropump.

\section{Design solutions of micropumps}

Following the design, hydraulic, and material challenges path, detailed solutions for the units and systems of the micropump were developed and assembled. The detailed design solutions of the micropump are shown in Fig. 6.

The pump features a system of external involute gears (1) with the number of teeth $z_{1}=z_{2}=15$. That ensured the lack of undercutting at the tooth root and, thus, its greater strength. The gears were formed together with the shafts. The main meshing parameters and gear dimensions calculated on the basis of [26] are presented in Table 1. The table shows that despite the increased number of teeth, the dimensions of the gears are not very large.

The micropump features the body (2) in the shape of a square prism. The body of such a shape was applied, because it is more compact and has smaller dimensions than the oval or rectangular bodies. Within the square cross-section, it is also easier to arrange the pins and bolts connecting three plates of the micropump as shown in Fig. 6. 
Fig. 6 The design of the gear micropump from plastics $q=0.28 \mathrm{~cm} 3 / \mathrm{rev}: 1$ - subsystem of the gears; 2 - subsystem of the body
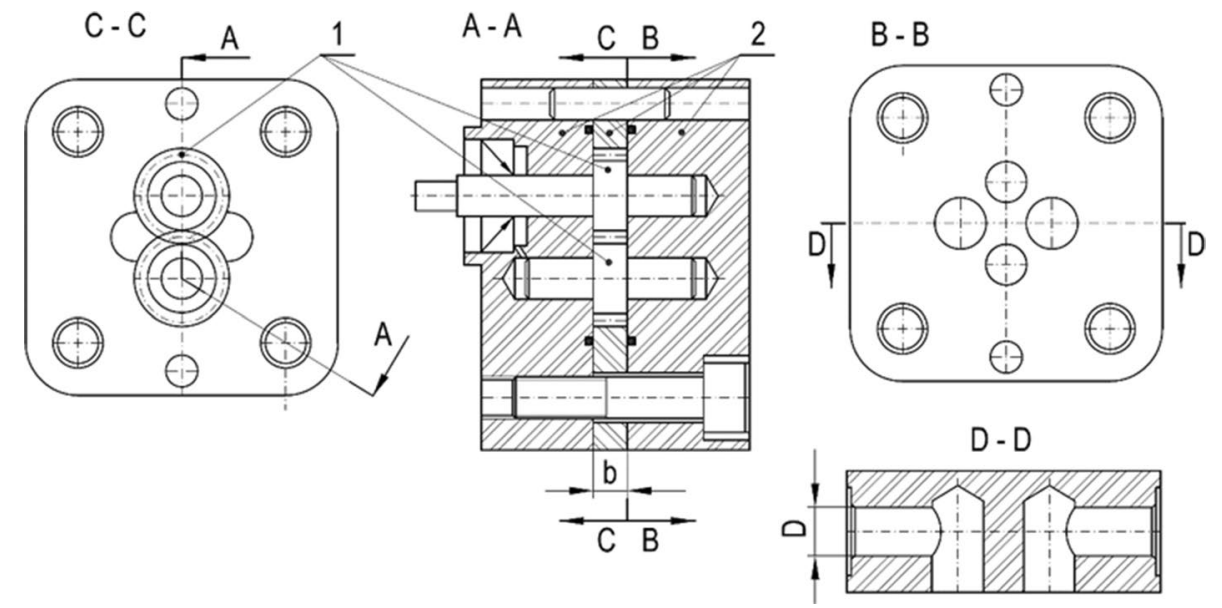

Table 1 Parameters of the involute mesh of the micropump

\begin{tabular}{lllll}
\hline Position & Parameter of gearing & Symbol & Value & Unit \\
\hline 1 & Gear profile & Involute & - & - \\
2 & Angle of the action & $\alpha$ & $20^{\circ}$ & $\mathrm{deg}$ \\
3 & Number of teeth & $z_{l}=z_{2}$ & 15 & - \\
4 & Modulus & $m$ & 0.8 & $\mathrm{~mm}$ \\
5 & Involute gear tooth & $y_{1}=y_{2}\left(h^{*}{ }_{a l}=h^{*}{ }_{a 2}\right)$ & 1 & - \\
& $\quad$ depth ratio & & & \\
6 & Involute gear outline & $x_{I}=x_{2}$ & 0 & - \\
7 & $\quad$ shift ratio & & & \\
8 & Addendum diameter & $d_{a}$ & 13.6 & $\mathrm{~mm}$ \\
9 & Dedendum diameter & $d_{f}$ & 10 & $\mathrm{~mm}$ \\
10 & Pitch diameter & $d$ & 12 & $\mathrm{~mm}$ \\
11 & Centre distance & $a$ & 12 & $\mathrm{~mm}$ \\
\hline
\end{tabular}

Table 2 Hydraulic parameters of the micropump

\begin{tabular}{lllll}
\hline Position & Hydraulic parameter & Symbol & Value & Unit \\
\hline 1 & Delivery per one revolution & $q$ & 0.28 & $\mathrm{~cm}^{3} / \mathrm{rev}$ \\
2 & Theoretical delivery & $Q_{t}$ & 0.42 & $1 / \mathrm{min}$ \\
3 & Delivery pulsation & $\delta$ & 18 & $\%$ \\
4 & Rotational speed & $n$ & $1500-2000$ & $\mathrm{rpm}$ \\
\hline
\end{tabular}

The pump body is divided into three parts. In the central part, there is a system of gears. The gear shafts are mounted directly in the bearing seats located in the side bodies, which eliminates expensive micro bearings. The three body parts are bolted together. Because the width $B$ of the central body was too small in relation to the diameter $D$ of the connection hole, an angular arrangement of the internal channels was used. That simultaneously allowed the enlargement of the cross-sections of the channels as well as of the inlet and outlet chambers and ensured the unrestricted inflow and outflow of the working medium to and from the system of the rotating gears. The central body was associated with the gear system in such a way that a minimum axial clearance $G_{A}=0.01-0.02 \mathrm{~mm}$ and a radial clearance $G_{R}=0.04-0.05 \mathrm{~mm}$ were obtained. The minimization of the radial clearance should ensure the internal tightness of the micropump and its higher hydraulic parameters. Assuming the value of the meshing parameters according to Table 1 , the unit-specific delivery $q=0.28 \mathrm{~cm}^{3} / \mathrm{rev}$ is derived from formula (4). However, formula (6) for the same parameters determines the value of the pulsation of delivery coefficient $\delta \approx 18 \%$.

This small value of the coefficient $\delta$ results from the application of an increased number of teeth $z_{1}=z_{2}=15$. Values of the hydraulic parameters are presented in Table 2 . Gears manufactured together with shafts will be made of
Table 3 Comparison of technical parameters of steel and plastics

\begin{tabular}{lllllll}
\hline Position & Technical parameter & Symbol & Unit & Steel & PBBK CF30 & PPS GF40 \\
\hline 1 & Yield strength & $R_{e}$ & $\mathrm{MPa}$ & 1000 & 250 & 83 \\
2 & Yield strength & $R e_{0,1}$ & $\mathrm{MPa}$ & 1000 & 160 & 65 \\
3 & Young modulus & $E$ & $\mathrm{MPa}$ & 210,000 & 13,000 & 6500 \\
4 & High temperature & $t$ & ${ }^{\circ} \mathrm{C}$ & 500 & 330 & 260 \\
5 & Shrinkage & $S$ & $\mathrm{~cm} / \mathrm{cm}$ & - & 0.005 & 0.005 \\
6 & Linear elongation & $W$ & $\mathrm{~cm} / \mathrm{K}$ & 0.00001 & 0.00004 & 0.00005 \\
7 & Water absorption & $A$ & $\%$ & - & $0.02-0.06$ & 0.01 \\
\hline
\end{tabular}


three different materials: constructional steel, PEEK CF 30, and PPS GF40 plastics. The properties of the materials are shown in Table 3.

Three systems of gears were designed:

- steel gear system (active and passive gear made of steel), - steel-plastic gear system (active gear-steel, passive gear-PEEK CF30),

- steel-plastic gear system (active gear-steel, passive gear-PPS GF40).

The utilization of the three gear systems is aimed at the exploration of the possibility of co-operation between the active and the passive gear and between the gear system and the plastic body of the pump. At the same time, it is possible to verify to what extent the plastic fulfils the postulate of high strength. PEEK GF30 was selected for the pump body. As Table 3 demonstrates, this material meets the strength requirements in a higher range, is dimensionally stable, and can be machined, which is important when making prototypes. The value of the 'reduced' elastic limit of $R_{\mathrm{e} 0,1}=160 \mathrm{MPa}$ is also very important. It is relatively large, which means that the body can be loaded with high mechanical and hydraulic loads, yet it will work in the elastic range.

\section{Strength analysis of the micropumps using the finite element method (FEM)}

The adopted design solution of the micropump was verified in the aspect of its strength. The effect of the mechanical load (the torque $T$, screw forces $Q$, and the intertooth forces $F$ ) and the hydraulic load (the operating pressure $p$ ) on the stress and deformation of the micropump body should be determined. As a result of the strain analysis, it is necessary to determine the places of the formation and the values of the clearance increase $\Delta G_{A}$ and $\Delta G_{R}$, which are critical determinants of the internal tightness of the pump and, consequently, its pressure $p$ and efficiency $\eta_{v}$ [see formulas (7), (8)]. However, as a result of the stress analysis, it should be determined whether they remain below the $R_{\mathrm{e} 0.1}$ limit and whether the pump housing will work in the elastic range (see Fig. 5).

Consequently, the range of anticipated loads on the micropump should be determined.

The geometrical model together with the arrangement of loads and the manufacturing method are shown in Fig. 7.

The mechanical loads (marked in Fig. 7 with arrows) are generated by the torque $T$ working on the pump's shaft and by the clamp $Q$ of the screws acting on the body. The torque generates intertooth forces $F_{i 1}$ and $F_{i 2}$ which load the shafts. At the same time, the gears and the body are worked on by the hydraulic load in a form of pressure $p$ (marked in Fig. 7 with the plus symbol). It generates forces $F_{p 1}$ and $F_{p 2}$ loading the gears. The summary of $F_{i 1}, F_{p 1}$ and $F_{i 2}, F_{p 2}$ results in the generating of forces $F_{s 1}, F_{s 2}$ which load the gears and the shafts of the pump. Forces $F_{s 1}$, and $F_{s 2}$, however, generate shafts' thrust trough $p_{b 1}, p_{b 2}$ working on the inner surface of the central body (2). The torque $T$ applied on the pump shaft is calculated from the formula:

$T=\frac{q}{2 \pi} \Delta p$,

where: $q$-pump delivery per 1 revolution of the shaft, $\Delta p-$ outlet pressure from the pump.

Intertooth forces $F_{i 1}, F_{i 2}$ are calculated according to [30] from the formula:
Fig. 7 Geometrical model of the gear micropump with the mechanical and hydraulic loads and restrains

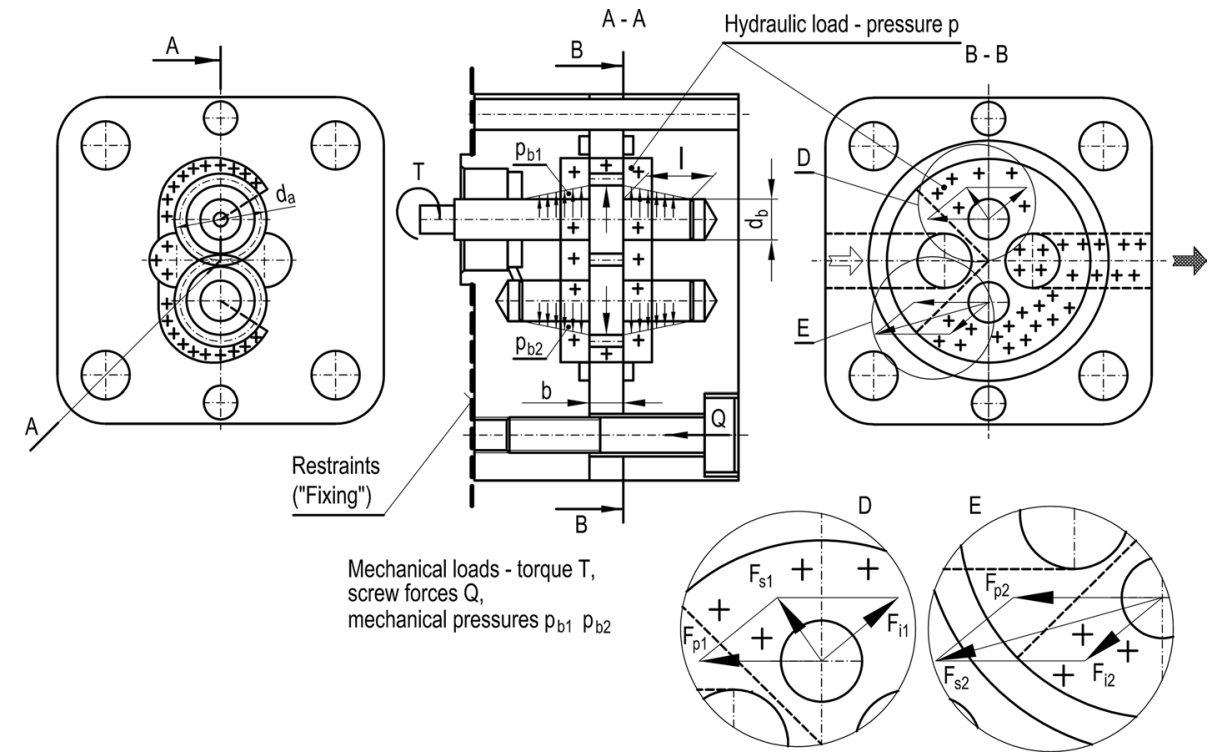


$F_{i 1}=F_{i 2}=\frac{2 T}{d \times \cos \alpha}$,

where: $d$-pitch diameter and $\alpha$-angle of the action.

Pressure generated forces $F_{p 1}, F_{p 2}$, are calculated from the formula:

$F_{p 1}=F_{p 2}=p * d_{a} * b$,

where: $d_{a}$-outside diameter of the gear and $b$-gear width.

As it has been mentioned above, the hydraulic loads are generated by pressure $p$ produced by the pump. The pressure acts on the central, back, and front bodies: in the central body, it works on the ports in which gears rotate, as well as on the chambers and the outlet from the pump. Pressure $p$ works also on the surface between the body and the shafts. The pressure distribution has been assumed following [30]. Pressure $p$ works also on the front areas of the front body (1) and of the back body (3), on the areas limited by the sealings.

Thrusts $p_{b 1}, p_{b 2}$ of the shafts on the central body are determined as:

$p_{b 1}=\frac{F_{s 1}}{2 * d_{b} * l}=\frac{F_{s 2}}{2 * d_{b} * l}$,

where: $d_{b}$-shaft diameter and $l$-shaft length.

Mechanical loads generated by force $Q$ of the screw clamp are determined as:

$Q=\frac{M}{0.5 * d_{s} * \operatorname{tg}(I l+D \rho \prime)}$,

where: $M$-screw torque, $d_{s}$, - thread diameter, $\gamma$-helix angle, and $\rho$ '-apparent friction angle.

The pump body has been restrained in accordance with the method of its assembling in practice, i.e., on the front surface of the front body. This is indicated in Fig. 7 by a dashed line.

A micropump with the PEEK CF30 body and steel gears were subject to strength tests. The pump was loaded with torque $T_{z}=0.5 \mathrm{Nm}$ and working pressure $p_{z}=5 \mathrm{MPa}$.

The calculation of the micropump was carried out using the Static Structural module of the ANSYS Workbench program. It is assumed that under the action of the pressure forces of the working fluid, relatively small movements of the structural elements occur, and the models of the materials used within the framework of the acting loads are linear elastic; therefore, linear analysis is used in the calculation (the Large Deflection option is disabled). Loading is carried out in one step. The choice of a direct or iterative solver is performed by the program automatically.

Figure 8 shows the results of strength tests of the micropump. Figure 8 a shows the deformations of the micropump body.
The figure shows that the pump body, especially the rear body, is deformed in the axial direction $a-a$. Axial clearance $\Delta G_{A}$ is formed between the rear body and the central body. Figure $8 \mathrm{~b}$ shows the deformation of the central body of the micropump. The central body is deformed in the radial direction $r-r$ and radial clearance $\Delta G_{R}$ is formed between the body and the outer diameter of the rotating gears. Deformation of the body in the axial $(a-a)$ and the radial $(r-r)$ directions and the formation of the $\Delta G_{A}$ and $\Delta G_{R}$ clearances result in a reduction of the pump's internal tightness and in a decrease in the value of the working pressure $p$ and volumetric efficiency $\eta_{v}$.

It was examined how much the $\Delta G_{A}$ and $\Delta G_{R}$ clearances change depending on the working pressure $p$, and on the material selected for the making of the pump body, i.e., steel, PEEK CF30, and PPS GF40 (see Table 3). The results are shown in Fig. 9. The figure demonstrates the same trend for all the considered materials where as pressure $p$ rises, axial clearances $\Delta G_{A}$ and radial clearances $\Delta G_{R}$ grow. The axial clearances $\Delta G_{A}$ are also larger than the radial clearances $\Delta G_{R}$.

At the same time, it is noted that the smallest clearances are obtained for the steel bodies, medium, for the PEEK CF30 bodies, and the largest, for the bodies made of PPS GF40. Finally, the FEM strength test showed that in the micropump made of PEEK CF30 at the working pressure $p_{e}=5 \mathrm{MPa}$, axial clearances $\Delta G_{A}=0.02 \mathrm{~mm}$ and radial clearances $\Delta G_{R}=0.01 \mathrm{~mm}$ can be expected. According to the formulas (7), (8) $G_{A}$ and $G_{R}$ assembly clearances (chapter 3) were added to the clearances $\Delta \mathrm{G}_{\mathrm{A}}$ and $\Delta \mathrm{G}_{\mathrm{R}}$ resulting from the deformation of the body (Fig. 8). The obtained values were compared with the allowable clearances, which, according to [30], are assumed as $G_{A \text { allow }}=0.05 \mathrm{~mm}$ and $G_{R \text { allow }}=0.1 \mathrm{~mm}$. As a result:

$G_{A}+\Delta G_{A}<G_{A \text { allow }}$

(0.03-0.04) $\mathrm{mm}<0.05 \mathrm{~mm}$

$G_{R}+\Delta G_{R}<G_{R \text { allow }}$

(0.05-0.06) $\mathrm{mm}<0.1 \mathrm{~mm}$.

On the basis of the conducted analyses, it can be predicted that the body of the micropump will be deformed, but within the allowable range which allows its functioning.

The stress analysis conducted on the pump bodies showed that the stresses were generated within the elastic stress range. For the body made of PEEK CF30, the stresses were:

$\sigma_{\max }=30 \mathrm{MPa}<R_{0,1}=160 \mathrm{MPa}$.

Therefore, it can be expected that the pump body made of PEEK CF30, during the loading-unloading cycle, is periodically deformed and $\Delta G_{A}$ and $\Delta G_{R}$ clearances are created, but it returns to its original shape and the clearances disappear. 
Fig. 8 Strengths and deformations of the micropump body designed from PEEK CF 30; torque $T=0.5 \mathrm{Nm}$, pressure $p=5 \mathrm{MPa}$. a Axial deformations and axial clearances increase $\Delta G_{A}$. b Radial deformations and radial clearances increase $\Delta G_{R}$ (a)

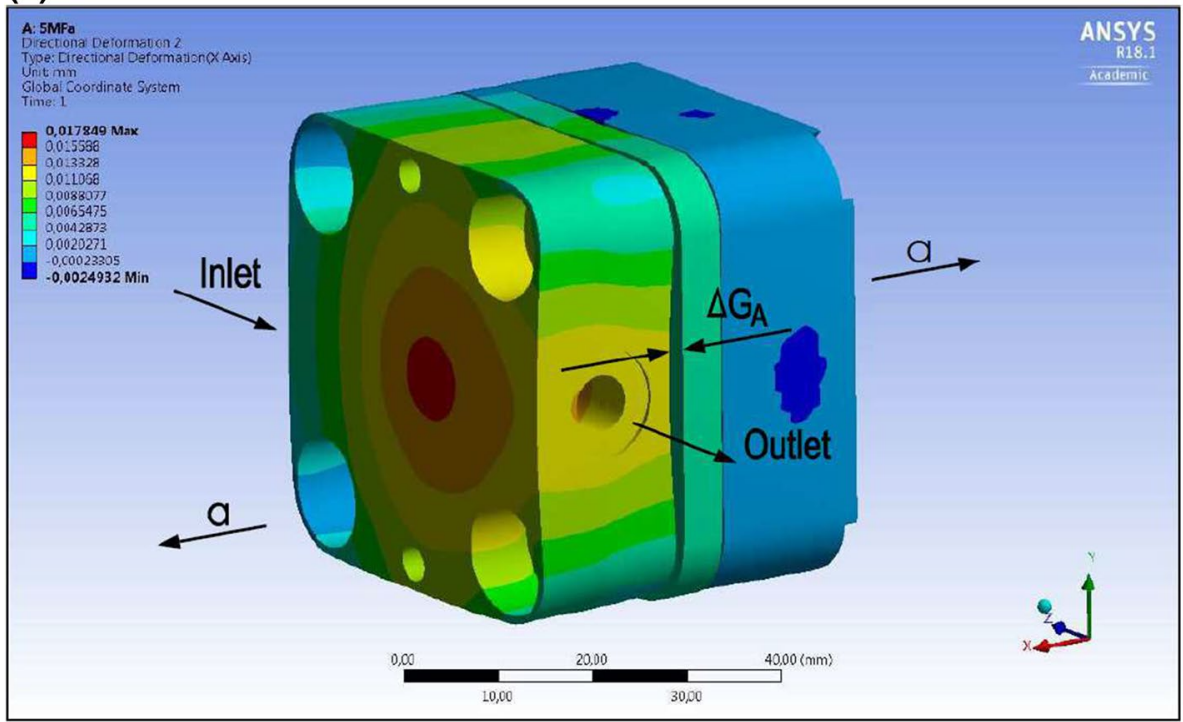

(b)

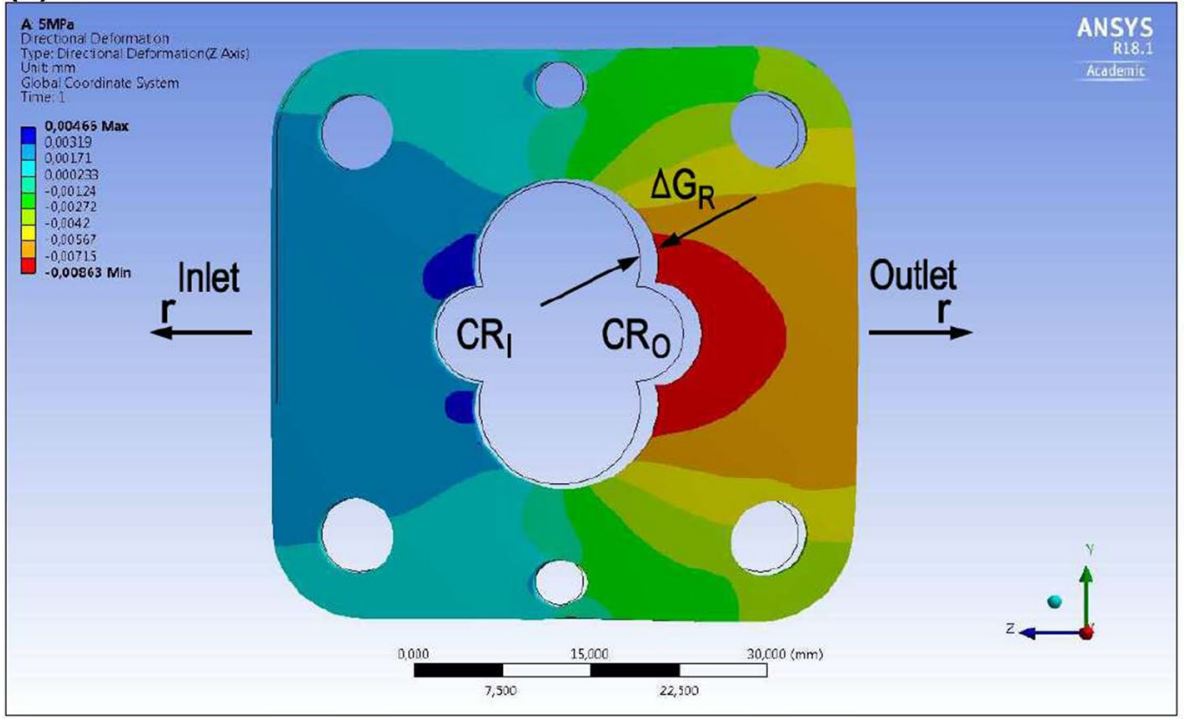

\section{The manufacture and experimental verification of the micropump model}

Using the design solution shown in Fig. 6, which was positively verified in terms of strength, a prototype of the micropump was made (see Fig. 10).

According to the assumptions, the body (2) of the micropump is made of PEEK CF30 (Fig. 10a), and the gear system (1) together with the shafts: steel, steel and plastic PEEK CF30, and steel and plastic PPS GF40 (Fig. 10b). All the pump units were made by machining. The pump was mounted using pins and bolted (4). Then it was placed on a test stand, the diagram of which is presented in Fig. 11.

The volumetric efficiency of the micropump was tested with the gear systems listed above, and the results are shown in Fig. 12. The body of the pump proved to co-operate correctly with all the gear systems. The highest values of the technical parameters were obtained for the system of the most durable and rigid steel gears. They worked in the range of working pressure up to $p=2.4 \mathrm{MPa}$ with the volumetric efficiency of $\eta_{v}=90-30 \%$. Lower parameter values were obtained at the 'mixed' gear assemblies featuring lower strength and rigidity. Working pressure $p=1.5 \mathrm{MPa}$ was generated and volumetric efficiency $\eta_{v}$, was lower by about $15 \%$ than the efficiency obtained for the steel gears.

When comparing the results of the theoretical analysis and the experimental research, discrepancies are visible. The results of the theoretical analysis suggested that the micropump can operate at pressures in the range of $5 \mathrm{MPa}$, because the allowable axial and radial clearances will be maintained by volume $\eta_{v}=30 \%$. The reason for obtaining lower parameters may be uncontrolled deformations and 
(a)

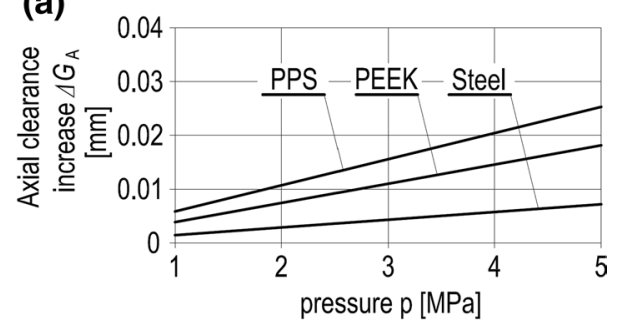

(b)

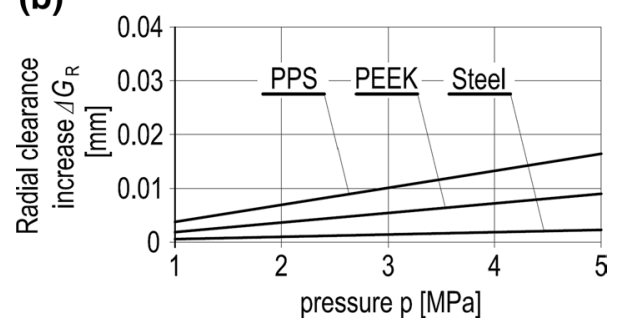

Fig. 9 Diagram of clearances in the micropump body made from steel, PEEK, and PPS. a Axial clearances increase $\Delta G_{A}$. b Radial clearances increase $\Delta G_{R}$

displacement of gears. This results in the generating of the uncontrolled internal clearances and increased volumetric losses. That is confirmed by the lower values of the parameters obtained for the more flexible gears made of PEEK and PPS compared to the more rigid steel gears (Fig. 12).

The micropump operated within the given parameters for $100 \mathrm{~h}$. In the first phase of the tests, plastic gears were used, which did not cause wear on the surface of the side body cooperating with the side surfaces of the gears. In the second phase of the tests, steel gears were used, which caused visible signs of wear on the surfaces of the side bodies.

It was also found that the co-operation of the 'hard' steel gears with the 'soft' PEEK body poses the risk of rapid wear of the face surface of the side bodies of the pumps.
Signs of such wear are depicted by Fig. 13b which shows that the gears have 'rings' pressed on the surface of the side body. That leads to the conclusion that from a tribological point of view, it is better to combine the plastic body with plastic gears.

Acoustic tests of the micropump with the PEEK body and the steel-steel and steel-PEEK system of gears were carried out. The results of the tests are presented in Fig. 14.

The main conclusion resulting from those tests is that the application of the steel-PEEK gear system in the micropump reduces the noise level compared to the pump featuring the steel-steel gear system. The figure also shows clearly that there is an increase in the pump noise level as the shaft speed increases.

\section{Summary and conclusions}

The paper presents an innovative process of designing a plastic gear micropump. This process brings new challenges in the fields of design, hydraulic, and material science.

The design challenges show that a pump ought to be built with as small dimensions and weight as possible but, simultaneously, high strength. For that purpose:

- gears with a small module $m<1$ and number of teeth $z_{1}=z_{2}=15>z_{g}$, which ensures no necessity of the undercut at the root of the teeth and an increased bending strength,

- the bolted body of the pump, in the shape of a prism with a square cross-section, which features small deformations and, as a result, small axial clearance $G_{A}$ and radial clearance $G_{R}$.
Fig. 10 The prototype of the gear micropump. a Overview of the pump: 1-subsystem of gears, 2-body, 3 - subsystem of the channels, and 4-connections. b Subsystem of the gears made from steel. c Subsystem of the gears made from plastics

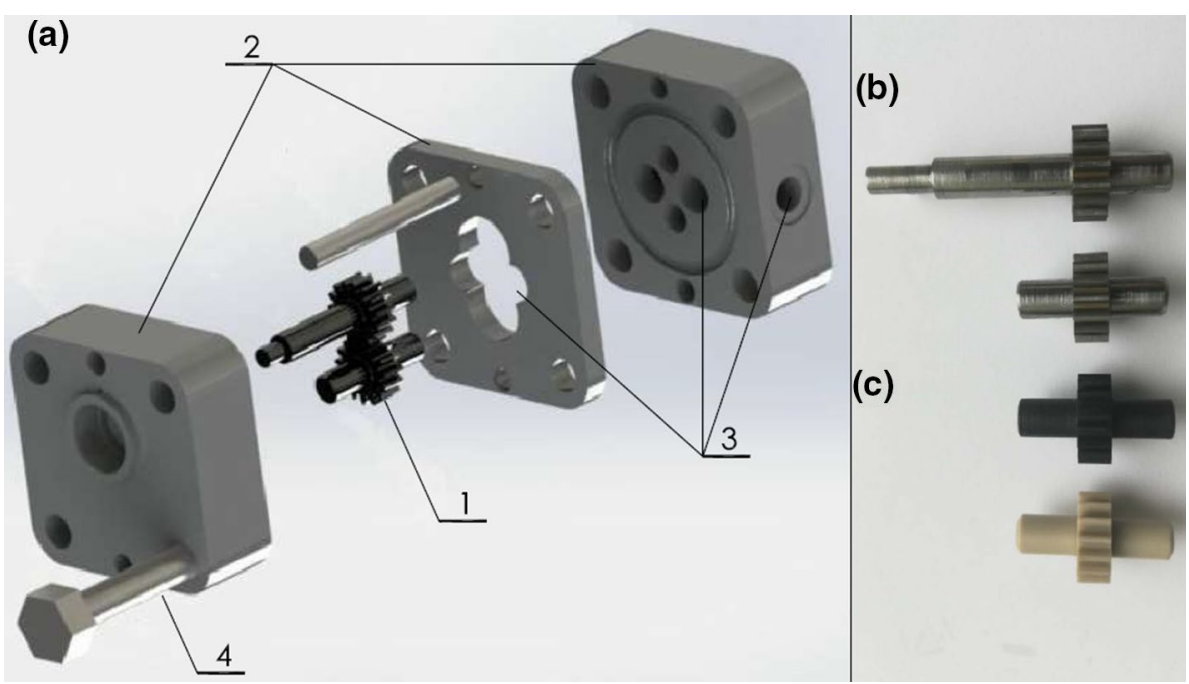


Fig. 11 Experimental stand for the examination of the micropump: a Scheme of the stand. $\mathbf{b}$ Table with the stands elements and their parameters (a)

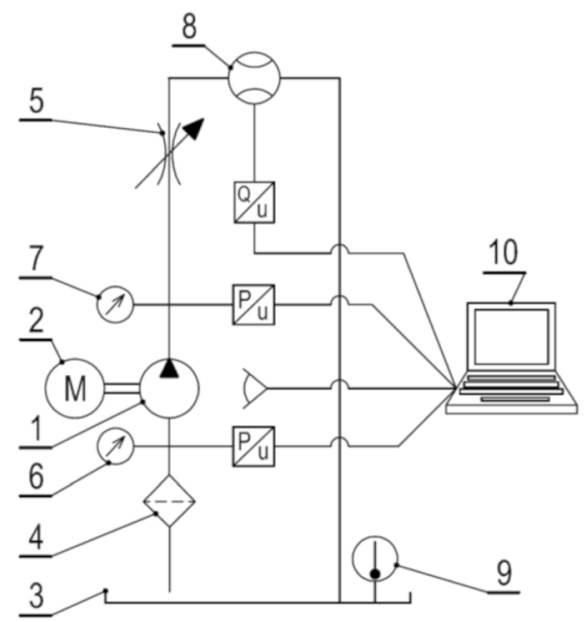

(b)

\begin{tabular}{|c|c|c|c|c|}
\hline \multirow{2}{*}{ No. } & \multirow{2}{*}{ Name } & \multicolumn{3}{|c|}{ Technical parameters } \\
\hline & & Type [unit] & value & accuracy \\
\hline 1 & Micropump & Delivery $[1 / \mathrm{min}]$ & $0.3 \div 0.6$ & - \\
\hline 2 & Electric motor & Power $[\mathrm{kW}]$ & 11 & - \\
\hline 3 & Tank & Volume [1] & 5 & - \\
\hline \multirow{2}{*}{4} & \multirow{2}{*}{ Filter } & Flow Rate [1/min] & 20 & - \\
\hline & & Size mesh $[\mu \mathrm{m}]$ & 25 & - \\
\hline 5 & Throttle valve & Response pressure [bar] & 50 & - \\
\hline 6 & Pressure transducer & Pressure [bar] & $0 \div 4$ & $\pm 0.4 \%$ \\
\hline 7 & Pressure transducer & Pressure [bar] & $0 \div 400$ & $\leq \pm 0.25 \%$ FS typ. \\
\hline 8 & Flow meter & Flow rate $[1 / \mathrm{min}]$ & $0.25 \div 8$ & $\pm 2.5 \%$ \\
\hline 9 & Temperature transducer & Temperature $\left[{ }^{0} \mathrm{C}\right]$ & $\begin{array}{c}- \\
25 \div 100\end{array}$ & $\leq \pm 1.5 \%$ FS typ. \\
\hline 10 & Computer & CPU frequency [M0hz] & $2 \times 2.3$ & - \\
\hline
\end{tabular}

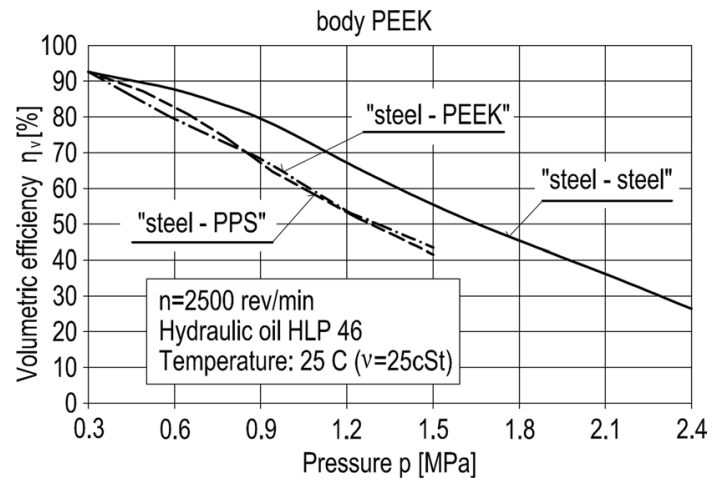

Fig. 12 The diagram of the volumetric efficiency of the micropump

The hydraulic challenges are related to ensuring low unitspecific delivery $q$ and low delivery rate pulsation $\delta$ while generating the highest possible pressure $p$ and volumetric efficiency $\eta_{v}$. (a)

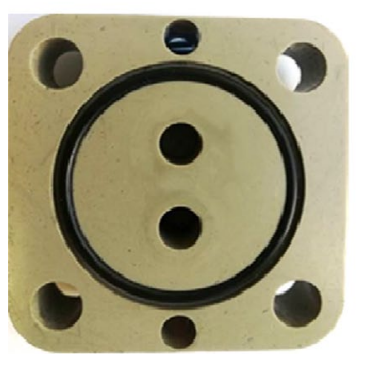

(b) wear

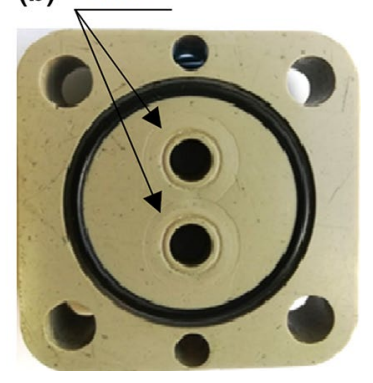

Fig. 13 View of the body surface co-operating with the gears. a Before the experimental research. b After the experimental research

Material challenges consist in the selection of a highstrength plastic and the dimensional stability capable of operating under cyclic loading and unloading conditions as well as at higher operating temperatures. 


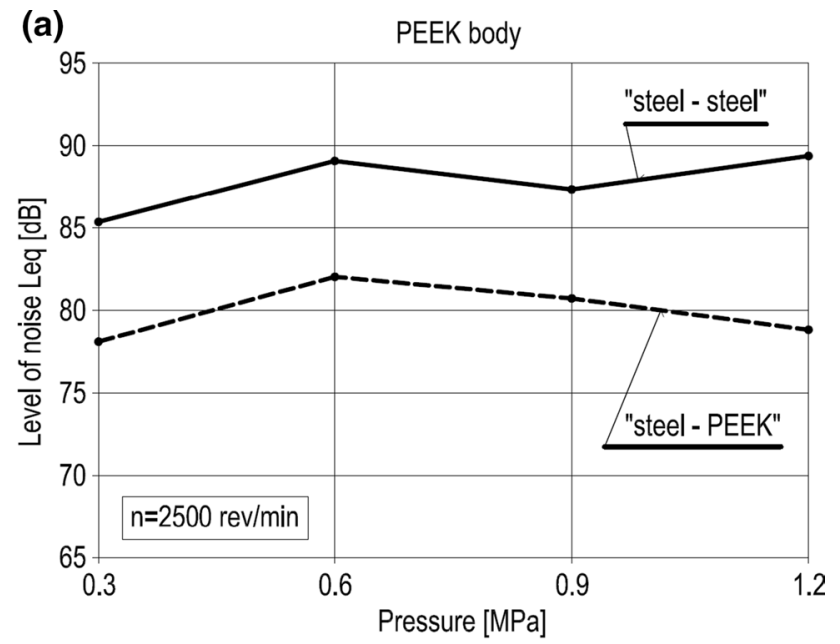

(b)

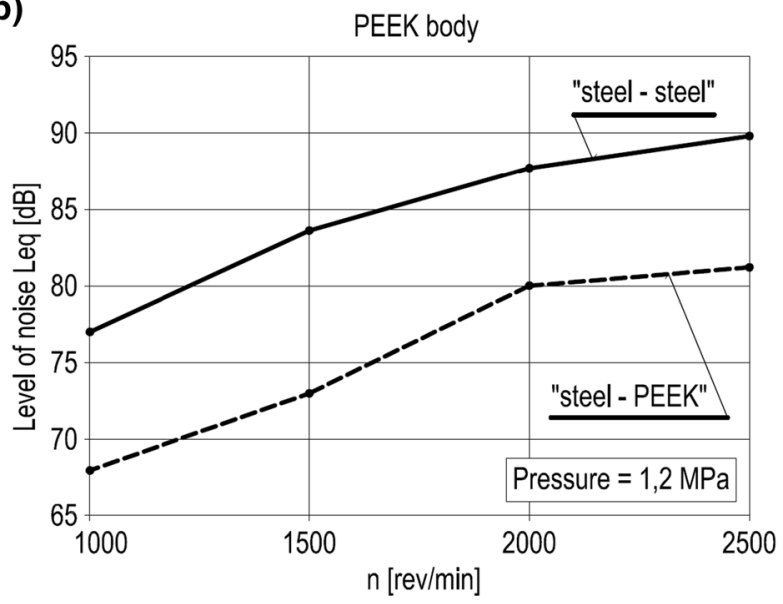

Fig. 14 Acoustic characteristics of the micropump with a PEEK body and gear systems: steel/steel and steel/PEEK. a Noise level depending on operating pressure Leq $=f(p)$. b Noise level depending on speed Leq $=f(n)$

Considering those challenges, a gear pump with a PEEK body and three interchangeable gear systems (steel-steel, steel-PEEK, and steel-PPS) were designed.

The strength analysis carried out by the FEM method showed that under the pressure load $p=5$, MPa increases in the axial and radial clearance in the micropump, but that increase in combination with the assembly clearance did not exceed the allowable values adopted for [30]:

$$
\begin{aligned}
& G_{A}+\Delta G_{A}<G_{A \text { allow }}=0.05 \\
& G_{R}+\Delta G_{R}<G_{R \text { allow }}=0.1,
\end{aligned}
$$

which enabled the proper performance of the micropump. At the same time, the stresses in the pump body are lower than the allowable stresses for PEEK which ensured the operation of the micropump body in the elastic range.

According to the design, a gear micropump featuring the delivery of $q=0.28 \mathrm{~m}^{3} / \mathrm{rev}$ was made, which successfully passed the experimental tests. The most advantageous technical parameters were obtained by the micropump with a PEEK body and a steel-steel gear system. It worked in the pressure range of $p=2.4 \mathrm{MPa}$ at the efficiency of $\eta_{\nu}=90 \div 30 \%$ for $100 \mathrm{~h}$. In the first phase of the tests, plastic gears were used, which did not cause wear on the surface of the side body co-operating with the side surfaces of the gears. In the second phase of the tests, steel gears were applied, which caused visible signs of wear on the surface of the side bodies. That leads to the conclusion that in the course of further development of the micropump, other material sets for the body and gears should be searched for to eliminate possible wear. For example, it could be PEEK, for the making of the gears, and PPS for the making of the body. Those materials will be sufficiently strong and rigid, and, at the same time, they will properly co-operate with each other, which was proved in $[9,12]$. Simultaneously, the material costs of the micropump will be reduced as the use of the expensive PEEK material will be limited. Moreover, the obtained results in volumetric efficiency of steel/PPS and steel/PEEK combination are almost similar. Therefore, a further analysis is necessary to explain these results.

The overall conclusion of the entire project is positive. It can be stated that making a micropump from plastics is possible and justified. The obtained working pressure and efficiency make it possible to classify the micropump as a unit for low-pressure hydraulic systems. The pressure, however, is higher than those used in the pneumatic systems. Micropumps and hydraulic microsystems made of plastics can, therefore, be competition for pneumatic microsystems.

At the same time, it is noted that there is still room for improvement of the design solution of the micropump, for example by modifying the shape of the body, screw connections or the optimization of the system of internal channels and clearances, as well as the application of other plastics.

Comparing designing process of gear pumps from metal and plastics, it can be noticed that many stages and designing methods are common. It applies i.a. designing of gearing, determination of technical parameters, shaping of the channels, and internal gaps. However, there are special stages of designing pumps from plastics, which concern choosing the plastic materials and strength analysis, especially analysis strains of the pump's body.

Acknowledgements The research was supported by the Ministry of Science and Education of the Russian Federation (Grants № 07772017-0017 and № 0777-2020-0015) and partly was funded by RFBR, project number 19-31-90095.

Funding The research was supported by the Ministry of Science and Education of the Russian Federation (Grants № 0777-2017-0017 and № 0777-2020-0015) and partly was funded by RFBR, project number 19-31-90095. 


\section{Compliance with ethical standards}

Conflict of interest The authors declare that they have no conflict of interest.

Ethical approval This article does not contain any studies with human participants or animals performed by any of the authors. It also has not been published by another journal.

Open Access This article is licensed under a Creative Commons Attribution 4.0 International License, which permits use, sharing, adaptation, distribution and reproduction in any medium or format, as long as you give appropriate credit to the original author(s) and the source, provide a link to the Creative Commons licence, and indicate if changes were made. The images or other third party material in this article are included in the article's Creative Commons licence, unless indicated otherwise in a credit line to the material. If material is not included in the article's Creative Commons licence and your intended use is not permitted by statutory regulation or exceeds the permitted use, you will need to obtain permission directly from the copyright holder. To view a copy of this licence, visit http://creativecommons.org/licenses/by/4.0/.

\section{References}

1. Liu Y, Schaefer JA. The-sliding friction of thermoplastic polymer composites tested at low speeds. Wear. 2006;261(5-6):568-77.

2. Schroeder R, Torres FW, Binde C, Klein AN, de Mello JDB. Failure mode in sliding wear of PEEK based composites. Wear Materials. 2013;301(1-2):717-26.

3. Chen B, Wang J, Yan F. Comparative investigation on the tribological behaviours of CF/PEEK composites under sea water lubrication. Tribol Int. 2012;52:170-7.

4. Wieleba W. Bezobsługowe łożyska ślizgowe z polimerów termoplastycznych. Oficyna Wydawnicza Politechniki Wrocławskiej, 2013.

5. Akaishi G, Kenji Doi Y, Matsushima IT, Fukuzaki Y. Production of high-carbon o:all and roller bearing steel in BOF-LF-CC process. Wire J Int. 1999;32(8):92-7.

6. Metalsi TF, Bourdim A. Stud of feasibility of plastic gear to reduce noise in a gear pump. Academic J. 2012;2(2):143-9.

7. Dearn K, Walton D. Acoustic emissions from polymeric gears, proceedings of the world congress on engineering, London, pp 1-3. (2009).

8. Hoskins TJ, Dearn KD, Kukureka SN, Walton D. Acoustic noise from polymer gears-a tribological investigation. Mater Des. 2011;32(6):3509-15.

9. Krawczyk J., Stryczek J. Designing of the gerotor pump body made of plastics, Proceedings of the ASME 2016 9th FPNI Ph.D Symposium on Fluid Power, 2016.

10. Stryczek J., Banaś M., Krawczyk J., Marciniak L., Stryczek P. The fluid power elements and systems made of plastics. Procedia Engineering 2016.

11. Mancini S., Neto A., Cioffi M., Carlos B. Replacement of metallic parts for polymer composite materials in motorcycle oil pumps. J Reinforced Plastics Composites, 2016.

12. Krawczyk J., Stryczek J. Construction and experimental research on plastic cycloidal gears used in gerotor pumps. Proceedings of the 8th FPNI Ph. D Symposium on Fluid Power, Lappeepranta, Finland, (2014).
13. Stryczek J, Bednarczyk S, Biernacki K. Geotor pump with pom gears: design, production technology. Res Arch Civil Mech Eng. 2014;14:391-7.

14. Rodionov L, Rekadze P. Exploration of acoustic characteristics of gear pumps with polymeric pinion shafts. J Proc Eng. 2015;106:36-45.

15. Marciniak L., Banaś, M., Stryczek J. The design and theoretical and experimental study of the plastic hydraulic valves. Proceedings of the ASME 2016 9th FPNI Ph.D Symposium on Fluid Power, 2016.

16. Banaś M., Antoniak P., Marciniak L., Stryczek J. Visualisation of flow phenomena in hydraulic throttle valves of plastic. Proceedings of the 14th International Conference on Vibration Engineering and Technology of Machinery (VETOMAC XIV), Lisbon, 2018.

17. Stryczek P., Przystupa F., Banaś M. Research on series of hydraulic cylinders made of plastics. Proceedings of Global Fluid Power Society PhD Symposium, Samara, 2018.

18. Harnisch M. Kunsttoffe in fluidtechnischen Antrieben. Oelhydraulik and Pneumatik, 2013.

19. Bonnano A. Materialli polimeriei applicaziani. Oleodynamica Pneumatica, 2008.

20. Gamez-Montero P.J., Antoniak P., Castilla R., Freire J., Krawczyk J., Stryczek J., Codina E. Magnet-sleeve-sealed mini trochoidalgear pomp prototype with polymer compozite gear. Energies, 2017.

21. Kollek W, Osiński P, Stosiak M, Wilczyński A, Cichoń P. Problems relating to high-pressure gear micropumps. Arch Civil Mech Eng. 2013;14(1):88-95.

22. Kollek W., Radziwanowska U. Energetic efficiency of gear micropumps, Arch Civil Mech Eng. 2015.

23. Rodionov L, Rekadze P, Stryczek J. A gear micropump without bearings production. Appl Mech Mater. 2015;775:352-6.

24. Rundo M. Models for flow rate simulationin gear pumps. A reviev. Energies. 2017;10:1261.

25. Castilla R., Gamez-Montero PJ., Raush G., Codina E. Method for fluid flow simulation of a gerotor pump using OpenFOAM J. Fluid Eng, 2017, 139.

26. Pellegri M, Manne V, Vacca A. A simulation model of Gerotor pumps considering fluid-structure interaction effects: formulation and validation. Mech Syst Signal Proc. 2020. https://doi. org/10.1016/j.ymssp.2020.106720.

27. Shakhmatov E.V., Prokofiev A.B, Kruchkov A.N, Makaryants G.M. The instability of the pipeline due to transporting fluid's pressure ripples. Proceedings of the 28th Congress of the International Council of the Aeronautical Sciences, Brisbane, 2012.

28. Prokofiev A.B., Makariyants G.M., Shakhmatov E.V. Modeling of Pipeline Vibration under the Pressure Ripples in the Working Fluid, Proceedings of the 17th International Congress on Sound \& Vibration, Cairo, 2010.

29. Rodionov L. and Rekadze P. A Gear Pump With Plastic Gears Noise And Vibration Research, Proceedings of the 22nd International Congress on Sound and Vibration, Florence, 2015.

30. Stryczek J. Fundamentals of designing hydraulic gear machines. Warszawa: Copyright by Wydawnictwo Naukowe PWN S.A; 2020.

31. Stryczek J., Biernacki K., Krawczyk J. Designing gear pump bodies using FEM Proceedings of the 14th International Scientific Conference: Computer Aided Engineering. Springer. 2019.

Publisher's Note Springer Nature remains neutral with regard to jurisdictional claims in published maps and institutional affiliations. 